\title{
Venous air embolus during prone cervical spine fusion: case report
}

\author{
Aurora S. Cruz, MD, MBA, ${ }^{1,5}$ Marc Moisi, MD, ${ }^{1}$ Jeni Page, NP, ${ }^{1}$ R. Shane Tubbs, PA-C, PhD, ${ }^{2}$ \\ David Paulson, MD, ${ }^{1}$ Michael Zwillman, MD, ${ }^{3}$ Rod Oskouian, MD, ${ }^{1}$ Arthur Lam, MD, ${ }^{4}$ and \\ David W. Newell, MD'
}

\begin{abstract}
Departments of ${ }^{1}$ Neurological Surgery and ${ }^{4}$ Anesthesia and Critical Care, Swedish Neuroscience Institute, Swedish Medical Center; ${ }^{2}$ Seattle Science Foundation, Seattle, Washington; ${ }^{3}$ Department of Anesthesia and Critical Care, The Methodist Hospital, Houston, Texas; and ${ }^{5}$ Department of Neurological Surgery, University of California, Irvine, Orange, California

Venous air embolism (VAE) is a known neurosurgical complication classically and most frequently occurring in patients undergoing posterior cranial fossa or cervical spine surgery in a sitting or semi-sitting position. The authors present a case of VAE that occurred during posterior cervical spine surgery in a patient in the prone position, a rare intraoperative complication. The patient was a 65-year-old man who was undergoing a C1-2 fusion for a nonunion of a Type II dens fracture and developed a VAE. While VAE in the prone position is uncommon, it is a neurosurgical complication that may have significant clinical implications both intraoperatively and postoperatively. The aim of this review is 2-fold: 1) to improve the general knowledge of this complication among surgeons and anesthesiologists who may not otherwise suspect air embolism in patients positioned prone for posterior cervical spine operations, and 2) to formulate preventive measures as well as a plan for prompt diagnosis and treatment should this complication occur.
\end{abstract}

http://thejns.org/doi/abs/10.3171/2016.5.SPINE16109

KEY WORDS venous air embolus; prone cervical fusion; prone surgery; C1-2 fusion

$\mathrm{V}$ ENOus air embolus (VAE) is a known but infrequent complication described in the literature.,13 The frequency of occurrence of VAE is higher in cranial procedures than in spine procedures, and the incidence is significantly higher among patients who undergo surgery in the seated position than in those who undergo surgery in the prone or supine position..$^{3,7}$ The incidence rate is between $20 \%$ and $40 \%$ in patients being operated on in the sitting position, depending on the study definitions and methods used to detect VAE. ${ }^{10,12}$ Venous air embolism occurs when air is introduced into the venous system in a low-pressure state. The air bubble, or embolus, is transported to the right atrium and then the right ventricle and eventually causes right ventricular outflow obstruction, with hemodynamic instability as a result.

We present a case of a VAE that occurred during posterior cervical spine surgery in a patient positioned prone.
Our aim is to highlight VAE as a differential diagnosis for patients undergoing posterior cervical spine surgery in the prone position. Through improvement of the general knowledge on this topic, the goal is for surgeons and anesthesiologists to understand possible anatomical pitfalls, institute preventive measures, implement treatment plans, and ultimately to improve patient outcomes and decrease morbidity and mortality for similar cases.

\section{Case Report}

This patient was a 65 -year-old man with a medical history of hypertension and rheumatoid arthritis who presented to clinic with the chief complaint of worsening neck pain, originally occurring after a car accident 35 years earlier. A CT scan was performed and revealed nonunion of a Type II dens fracture. Review of his medi-

ABBREVIATIONS CPR = cardiopulmonary resuscitation; TEE = transesophageal echocardiography; VAE = venous air embolism.

SUBMITTED January 20, 2016. ACCEPTED May 26, 2016.

INCLUDE WHEN CITING Published online July 22, 2016; DOI: 10.3171/2016.5.SPINE16109. 
cal record revealed CT evidence of this fracture as early as 2003, with nonunion noted at that time. A posterior C1-2 fusion procedure with an iliac crest bone graft and neuromonitoring (somatosensory and motor evoked potential monitoring) was scheduled. Following uneventful induction of general anesthesia, the patient was positioned prone with his head in a Mayfield head clamp. During the surgical exposure, the surgeon encountered substantial venous bleeding, which was controlled with bipolar electrocautery and hemostatic agents and placing the patient in a slight reverse-Trendelenburg position. Shortly after, with the placement of the second C-1 lateral mass screw, the patient had a precipitous drop in blood pressure as well as an abrupt decrease in end-tidal $\mathrm{CO}_{2}$ and pulse oximetry reading. Almost simultaneously, the neuromonitoring technician recognized that he had lost all evoked potential signals but suspected that it was a technical issue and did not notify the surgeon or the anesthesiologist. The attending anesthesiologist promptly notified the surgical team of the hypotension and decrease in end-tidal $\mathrm{CO}_{2}$. The patient's blood pressure was supported with administration of vasopressor agents (phenylephrine infusion and bolus of ephedrine), and the patient's airway was suctioned, which temporarily improved his oxygen saturation. As the end-tidal $\mathrm{CO}_{2}$ and blood pressure did not improve, the procedure was aborted, and the patient was turned supine, in anticipation of the possible need for cardiopulmonary resuscitation (CPR). Transesophageal echocardiography (TEE) was performed and revealed apical hypokinesis but no obvious evidence of air. This was thought to be consistent with possible passage of air into the right coronary artery, either from a patent foramen ovale, or via transpulmonary passage of air. The patient's blood pressure and oxygenation improved, but he continued to require infusion of vasopressors. After 15 minutes, it was decided that his condition was too unstable to allow for continuing the operation. Therefore, the wound was covered with a sterile dressing, and the patient was transferred directly to the neurointensive care unit with continued sedation and intubation. Postoperatively, over the next 2 days the patient was gradually weaned off his vasopressors and extubated with no neurological deficits. A subsequent cardiac workup did not reveal any myocardial damage, indirectly confirming that an air embolus was the likely etiology. The patient was discharged home, returned 2 weeks later, and underwent uneventful completion of the spine fusion procedure. Of note, invasive blood pressure monitoring was instituted before the second surgical procedure, and the operation was performed entirely with the patient in a strictly horizontal position without any head-up elevation.

\section{Discussion}

Venous air emboli occur when a bolus of air is forced into an open vein by way of atmospheric pressure exceeding venous pressure ${ }^{4,14}$ Clinically benign, asymptomatic venous air emboli occur frequently during any surgical procedure performed at a surgical site above the level of the heart and require no intervention. When significant volumes of air are introduced into the venous system, an accumulation of air into the right heart and pulmonary circulation may cause right ventricular outflow obstruction, with a dramatic fall in cardiac output manifested in a decline in blood pressure and end-tidal $\mathrm{CO}_{2}$ as well as oxygen saturation. However, these signs are relatively nonspecific, and the diagnosis of VAE is often not considered, particularly when the procedure or patient position is presumed to be associated with a low risk for VAE. Therefore, a high index of clinical suspicion of VAE is needed in order for practitioners to promptly diagnose this condition and initiate appropriate therapy.

The cervical venous anatomy is of particular interest in the context of posterior approaches to the cervical spine, and understanding this anatomy is important to understanding why VAE may occur. Cadaveric studies have consistently demonstrated the presence of anterior, lateral, and posterior vertebral venous plexuses at the craniocervical junction, most often draining intracranially through the marginal sinus. ${ }^{17}$ When approaching the posterior cervical spine, regardless of positioning, the posterior external vertebral plexus will be encountered first, as this plexus surrounds the posterior elements of the spine. The posterior external vertebral plexus drains to the superior vena cava via the vertebral, deep cervical, and jugular veins. ${ }^{9}$ It was recently shown that the external vertebral venous plexus differs from many of the other vertebral venous plexuses in that some of its veins have valves that direct flow toward the internal vertebral venous plexus. ${ }^{9}$ However, it is still believed that many of the veins of the vertebral venous plexuses are valveless, the venous pressure being solely dependent on position relative to the level of the heart. ${ }^{9}$ The dependence of pressure on patient positioning is consistent with the well-documented knowledge of VAE in the upright semi-sitting position. Knowledge of the venous anatomy, especially in the case of potential pathology, is important for avoidance and determining the location of venous bleeding during a posterior cervical approach in both the prone and semi-sitting positions. In addition, the venous plexus around the atlanto-axial junction may be enlarged compared with other levels, further increasing the risk of VAE. ${ }^{5}$

There are practical steps that may be adopted to help mitigate clinically relevant VAE or prevent it from occurring. ${ }^{12}$ Reports in the literature support changes in patient positioning through the modification of the traditional semi-sitting position to increase venous pressure and reduce the likelihood of air being pulled into the venous system. Raising the legs above the heart to improve venous return, periodic jugular massage, and keeping a higher positive end expiratory pressure (PEEP) ${ }^{12,18}$ are suggested as methods to increase venous pressure when using the semi-sitting position, although these are generally considered to be impractical. Intraoperative transcranial Doppler ultrasonography and preoperative examination with transesophageal echocardiography can be used to screen for the presence of a patent foramen ovale when use of a sitting positioning is contemplated, although there is little evidence from reports in the literature to support this level of screening in other surgical positions such as prone. ${ }^{10,16}$ Precordial Doppler monitoring during surgery is another method of screening, allowing for early detection and 
prompt subsequent management of VAE, but it is difficult to implement in the prone position. ${ }^{15}$

Early detection and appropriate treatment of VAE is critical for reducing morbidity and mortality associated with this complication. VAE is classically associated with tachypnea (in a spontaneously breathing patient) and a sudden decrease in end-tidal $\mathrm{CO}_{2}$ with oxygen desaturation and vital-sign instability in some cases. ${ }^{8,14}$ Pulmonary artery pressure rises as a result of strain on the heart from right ventricular outflow obstruction by the air embolus. ${ }^{14}$ The main treatment considerations in the treatment of VAE are preventing further entry of air into the venous system and removing the air embolus from the heart by improving its contractility. When VAE is suspected, reports in the literature recommend flooding the field with irrigation, sealing off venous bone bleeding with wax, moving the patient into a head-down or Trendelenburg position to increase venous pressure, applying jugular vein compression, and coagulating the venous bleeding using bipolar electrocautery.,6,14 Additionally, the use of an appropriately placed central venous line can be beneficial, allowing air to be directly aspirated from the right atrium, preventing further air entry. ${ }^{14}$ In the case of hypotension, inotropic agents should be used to improve contractility, reduce the effects of outflow obstruction from the VAE, ${ }^{14}$ and propel the air embolus into the pulmonary circulation. Good communication among members of the entire surgical team is crucial. As noted in our case, there was loss of evoked potential signals during the VAE episode, and had the surgical/anesthesia team been notified promptly, the diagnosis could conceivably have been made a few minutes earlier. As it happened, this slight delay did not lead to any adverse outcome, and the need for CPR never arose. Nonetheless, this illustrated the importance of clear communication in the operating room.

The rate of VAE in cervical spine cases is approximately $7 \%-8 \%$ in patients who undergo surgery in the sitting or semi-sitting position. ${ }^{5}$ In the setting of prone positioning, there are several reported cases of VAE during lumbar and thoracic laminectomy and fusions. ${ }^{2,11}$ Clinically significant VAE is rare in patients undergoing cervical surgery in the prone position, and only one case had been reported previously. ${ }^{5}$ The case reported by Dumont et al. is eerily similar to the one reported here. A patient who was positioned prone for atlantoaxial arthrodesis was placed in a slight reverse-Trendelenburg position, which the authors believe contributed to the negative venous pressure environment, as in our case. Unlike in our case, in the case reported by Dumont et al., the patient's hemodynamic instability led to asystole; the patient was turned over and CPR was performed before the vital signs returned to normal. TEE was performed intraoperatively and revealed "increased atrial pressures and a shifted atrial septum consistent with air embolus, although at this point no intracardiac air was noted." 5 The operation was then completed uneventfully, and the patient did not suffer any permanent cardiac or neurological deficits. The primary difference between the previously published case and the case presented in this paper was the severity of hemodynamic instability and the subsequent management. In our case, the patient's vital signs returned to normal without CPR, likely due to a less physiologically significant VAE, as demonstrated by subsequent TEE, which only revealed right atrial enlargement and decrease in systolic function. This also may have been a result of the longer interval between hemodynamic changes and TEE, as in the case reported by Dumont et al., intraoperative TEE was performed within 15 minutes of hemodynamic decline. We believe that, given the hemodynamic instability necessitating high-dose vasopressor therapy in our case, it was prudent to abort the surgical procedure and complete it at a later date.

\section{Conclusions}

Although rare, because of the anatomical variation of the venous plexus at the atlanto-axial junction, VAE in the prone position can be an unexpected surgical complication with significant clinical sequelae. Avoidance of a head-up position, a high index of suspicion, good communication among the surgical/anesthesia/monitoring team, and the willingness to abort the procedure are the necessary elements that will enhance patient safety and improve outcome.

\section{References}

1. Albin MS, Carroll RG, Maroon JC: Clinical considerations concerning detection of venous air embolism. Neurosurgery 3:380-384, 1978

2. Albin MS, Ritter RR, Pruett CE, Kalff K: Venous air embolism during lumbar laminectomy in the prone position: report of three cases. Anesth Analg 73:346-349, 1991

3. Ballantine RI: Anaesthetic advances in neurosurgery. Proc $\mathbf{R}$ Soc Med 63:821-825, 1970

4. Barth L: [Mechanism of air embolism in the area of the human cervical veins. 1. Effect of hydrostatic, respiratory, and cardiac factors on pressure in the v. cava cranialis and v. jugularis externa.] Anaesthesist 20:356-363, 1971 (Ger)

5. Dumont TM, Stockwell DW, Horgan MA: Venous air embolism: an unusual complication of atlantoaxial arthrodesis: case report. Spine (Phila Pa 1976) 35:E1238E1240, 2010

6. Gale T, Leslie K: Anaesthesia for neurosurgery in the sitting position. J Clin Neurosci 11:693-696, 2004

7. Ganslandt O, Merkel A, Schmitt H, Tzabazis A, Buchfelder M, Eyupoglu I, et al: The sitting position in neurosurgery: indications, complications and results. a single institution experience of 600 cases. Acta Neurochir (Wien) 155:18871893,2013

8. Gracia I, Fabregas N: Craniotomy in sitting position: anesthesiology management. Curr Opin Anaesthesiol 27:474-483, 2014

9. Griessenauer CJ, Raborn J, Foreman P, Shoja MM, Loukas M, Tubbs RS: Venous drainage of the spine and spinal cord: a comprehensive review of its history, embryology, anatomy, physiology, and pathology. Clin Anat 28:75-87, 2015

10. Hervías A, Valero R, Hurtado P, Gracia I, Perelló L, Tercero FJ, et al: [Detection of venous air embolism and patent foramen ovale in neurosurgery patients in sitting position.] Neurocirugia (Astur) 25:108-115, 2014 (Span)

11. Horlocker TT, Wedel DJ, Cucchiara RF: Venous air embolism during spinal instrumentation and fusion in the prone position. Anesth Analg 75:152-153, 1992

12. Jadik S, Wissing H, Friedrich K, Beck J, Seifert V, Raabe A: A standardized protocol for the prevention of clinically relevant venous air embolism during neurosurgical interventions in the semisitting position. Neurosurgery 64:533-539, 2009 
13. O'Higgins JW: Air embolism during neurosurgery. A case report. Br J Anaesth 42:459-462, 1970

14. Palmon SC, Moore LE, Lundberg J, Toung T: Venous air embolism: a review. J Clin Anesth 9:251-257, 1997

15. Schubert A, Deogaonkar A, Drummond JC: Precordial Doppler probe placement for optimal detection of venous air embolism during craniotomy. Anesth Analg 102:1543-1547, 2006

16. Stendel R, Gramm HJ, Schröder K, Lober C, Brock M: Transcranial Doppler ultrasonography as a screening technique for detection of a patent foramen ovale before surgery in the sitting position. Anesthesiology 93:971-975, 2000

17. Tubbs RS, Ammar K, Liechty P, Wellons JC III, Blount JP, Salter EG, et al: The marginal sinus. J Neurosurg 104:429_ 431, 2006

18. Voorhies RM, Fraser RA, Van Poznak A: Prevention of air embolism with positive end expiratory pressure. Neurosurgery 12:503-506, 1983

\section{Disclosures}

Dr. Moisi reports a consultant relationship with Synaptive Medical.

\section{Author Contributions}

Conception and design: Moisi. Acquisition of data: Moisi, Cruz. Analysis and interpretation of data: Moisi, Page. Drafting the article: Moisi, Cruz. Critically revising the article: Moisi, Page, Tubbs, Paulson, Zwillman, Oskouian, Lam, Newell. Reviewed submitted version of manuscript: Moisi, Tubbs. Study supervision: Oskouian, Lam, Newell.

\section{Correspondence}

Marc D. Moisi, Department of Neurological Surgery, Swedish Neuroscience Institute, 550 17th Ave., Seattle, WA 98122. email: moisimd@aol.com. 